\title{
Nervio Interóseo Anterior: Trayecto, Distribución e Implicancias Clínicas
}

\author{
Anterior Interosseous Nerve: Course, Distribution and Clinical Implications
}

Riveros, A. ${ }^{1,2}$; Olave, E. ${ }^{3}$ \& Sousa-Rodrigues, C. $^{4}$

RIVEROS, A.; OLAVE, E. \& SOUSA-RODRIGUES, C. Nervio interóseo anterior: Trayecto, distribución e implicancias clínicas. Int. J. Morphol., 36(3):1079-1086, 2018.

RESUMEN: El principal ramo del nervio mediano en el antebrazo es el nervio interóseo anterior (NIA), el cual inerva la mayoría de los músculos del plano profundo del compartimiento anterior del antebrazo. Existen diferentes descripciones sobre el punto de origen y su trayecto del NIA, así como también respecto a ramos comunicantes de este, con otros nervios del antebrazo. Algunas de estas descripciones concuerdan de que el NIA pueda atraparse en su tránsito por los arcos formados en las cabezas de origen de los músculos pronador redondo y flexor superficial de los dedos. El objetivo fue determinar el punto de origen, trayecto, músculos inervados y presencia de ramos comunicantes del NIA con otros nervios del antebrazo. Se utilizaron 30 antebrazos de cadáveres adultos brasileños fijados en formalina pertenecientes al laboratorio de anatomía de la UNCISAL, Maceió, Brasil. El punto de origen del NIA alcanzó un promedio de 46 mm con una desviación estándar de 17,54 mm, distal a la línea biepicondilar. En un $37 \%$ de los casos, este nervio se originó en el tramo existente entre los arcos musculares mencionados y en un $23 \%$ surgió proximal a la ubicación de estos. En todos los casos, el NIA inervó a los músculos flexor profundo de los dedos y pronador cuadrado y en un $93 \%$ también inervó al músculo flexor largo del pulgar. Un caso presentó ramo comunicante entre el NIA y el nervio ulnar. En el $10 \%$ de los casos, presentó la variante muscular cabeza accesoria del músculo flexor largo del pulgar. Todos estos hallazgos deben considerarse al momento de diagnosticar correctamente los diferentes síndromes de atrapamiento que afectan tanto al NIA como al nervio mediano en la región cubital y en el antebrazo. Así mismo, el éxito de procedimientos quirúrgicos de estas regiones está supeditado a un conocimiento detallado del trayecto y distribución de estos nervios.

PALABRAS CLAVE: Anatomía; Inervación; Nervio interóseo anterior; Ramo comunicante; Variaciones anatómicas.

\section{INTRODUCCIÓN}

La inervación de la mayoría de los músculos del compartimiento anterior del antebrazo se asocia al nervio mediano o su principal ramo denominado nervio interóseo anterior (NIA) (Nervus interosseus antebrachii anterior) (Comité Federativo sobre Terminología Anatómica \& Sociedad Anatómica Española, 2001). El origen de este nervio se describe desde la superficie posterior del nervio mediano, cuando éste transita entre las dos cabezas del músculo pronador redondo, lo cual sucede a unos 43 a $54 \mathrm{~mm}$ distal a una línea que une los dos epicóndilos del húmero, la cual se denomina línea biepicondilar (LBE) (Linell, 1921; Canovas et al., 1998). En este sentido, Llusá et al. (2013) plantearon que el NIA, se desprende del nervio mediano, en algunas oportunidades, a la altura de la arcada o arco que se forma cuando se unen las dos cabezas de origen del músculo pronador redondo, y en otras, cuando el nervio mediano pasa bajo la arcada que se forma en el origen del músculo flexor superficial de los dedos. Relacionado con ello, Dellon \& Mackinnon (1987) encontraron que, en 16 de 31 cadáveres analizados, el NIA se relacionó con algún arco fibroso que se formaba en el origen ya sea del músculo pronador redondo (56\%), del músculo flexor superficial de los dedos (19\%) o con $\operatorname{ambos}(25 \%)$.

Respecto a la inervación de los músculos profundos del compartimiento anterior del antebrazo, Llusá et al. señalaron que el nervio mediano no emite ramos directos a los músculos profundos del compartimiento anterior del antebrazo y en su reemplazo, el encargado de dicha inervación sería en forma exclusiva el NIA, el cual entrega una serie de ramos que abordan en su longitud a los músculos flexor profundo de los dedos y flexor largo del pulgar, además de otros

\footnotetext{
${ }^{1}$ Departamento de Ciencias Morfológicas, Facultad de Medicina y Ciencia, Universidad San Sebastián, Lientur 1457, Concepción 4080871, Chile.

${ }^{2}$ Programa de Doctorado en Ciencias Morfológicas, Universidad de La Frontera, Temuco, Chile.

${ }^{3}$ Facultad de Medicina, Universidad de La Frontera, Temuco, Chile.

${ }^{4}$ Universidad Estadual de Ciencias da Saude de Alagoas, Maceió, Brasil.
} 
destinados al músculo pronador cuadrado y a la membrana interósea (Nervio de Rauber), los ramos diafisiarios destinados para el perióstio del radio y de la ulna (Nervio de Lazorthes) (Rauber, 1898; Tountas \& Bergman, 1993; Khale et al., 1994; Williams et al., 1998; Moore \& Dalley, 2002; Drake et al., 2005; Schünke et al., 2005; Leversedge et al., 2010; Pró, 2012). Contrario a lo anteriormente descrito, Sappey (1889), Cruveilhier (1851), Testut \& Latarjet (1972), y Rouvière \& Delmas (2005) señalaron que existe un ramo del nervio mediano destinado a la capa muscular profunda de este compartimiento, el cual se divide en varios ramos, uno lateral, destinado al músculo flexor largo del pulgar, dos ramos mediales para la mitad lateral del músculo flexor profundo de los dedos y un ramo medio denominado NIA, el cual, en su trayecto entregaría pequeños ramos a los mencionados músculos para finalmente terminar inervando al músculo pronador cuadrado y la articulación radiocarpiana.

Sumado a los ramos descritos y en relación a la presencia de ramos comunicantes en el antebrazo, Roy et al. (2016) describieron la existencia de ramos que comunican al nervio mediano o al NIA con el nervio ulnar, conocida como de Martin-Gruber (unión descrita por el anatomista sueco, Martin en 1763 y complementada por Gruber en 1870), la que se reportó con una prevalencia que fluctuó entre el 10 al $30 \%$. Esta comunicación lleva fibras motoras y sensitivas desde el nervio mediano o el NIA, hacia el nervio ulnar, están destinadas a la musculatura intrínseca de la mano; generando que el nervio mediano esté vinculado con la inervación de las dos cabezas del músculo flexor corto del pulgar o del músculo aductor del pulgar (Llusá et al.; Pró) (Figs. 1A-C).
Al respecto, existen diferentes clasificaciones asociadas a los tipos de comunicaciones entre los nervios mediano, NIA y el nervio ulnar. La Tabla I resume lo propuesto por Nakashima (1993), versus lo planteado por RodriguezNiedenführ et al. (2002).

Otra variante anatómica que guarda relación con el potencial atrapamiento del nervio mediano o del NIA, es la presencia de una cabeza accesoria del músculo flexor largo del pulgar, conocida como fascículo o músculo de Gantzer (Leversedge et al.). Presente en un $45 \%$ de los antebrazos, esta cabeza con origen en el epicondilo medial del húmero, se suma al tercio medio del músculo y en su trayecto se dispone ventral al NIA (Dellon \& Mackinnon; Llusa et al.).

Basado en lo anterior, el propósito del presente estudio fue determinar el origen, trayecto, distribución y presencia de ramos comunicantes del NIA con otros nervios del antebrazo.

\section{MATERIAL Y MÉTODO}

Se utilizaron 30 miembros superiores (16 derechos y 14 izquierdos) de individuos adultos, brasileños, pertenecientes a la Facultad de Medicina de la Universidad Estadual de Ciencias da Saúde de Alagoas (UNCISAL), Maceió, Brasil. Estos cadáveres se encontraban fijados bajo inmersión en una solución con formaldehído al $10 \%$. Esta investigación se formuló bajo una metodología de carácter cuantitativo, no experimental, transeccional y descriptiva, para lo

Tabla I. Clasificación de ramos comunicantes entre nervios mediano, interóseo y ulnar

\begin{tabular}{|c|c|c|c|}
\hline Nakashima (1993) (n: 108). & $\begin{array}{l}\text { Porcentaje de } \\
\text { presencia de ramo } \\
\text { comunicante }\end{array}$ & Rodriguez-Niedenführ et al., (2002) (n:140). & $\begin{array}{l}\text { Porcentaje de } \\
\text { presencia de ramo } \\
\text { comunicante }\end{array}$ \\
\hline $\begin{array}{l}\text { Presencia de ramos comunicantes: } \\
\qquad 21 \% .\end{array}$ & & $\begin{array}{l}\text { Presencia de ramos comunicantes: } \\
\qquad 14 \% .\end{array}$ & \\
\hline $\begin{array}{l}\text { Tipo I A: Desde el nervio interóseo } \\
\text { anteri or hacia el nervio ulnar. }\end{array}$ & 57 & $\begin{array}{l}\text { Tipo I A: Desde el tronco común del nervio mediano } \\
\text { para los músculos superficiales del compartimiento } \\
\text { anterior, hacia el nervio ulnar. }\end{array}$ & 47 \\
\hline $\begin{array}{l}\text { Tipo I B: Desde el nervio mediano } \\
\text { hacia el nervio ulnar. }\end{array}$ & 4 & $\begin{array}{l}\text { Tipo I B: Origen en el nervio mediano, entre el origen } \\
\text { del tronco común del nervio mediano para los } \\
\text { músculos superficiales del compartimi ento anterior y } \\
\text { el origen del nervio interóseo anterior, hacia el nervio } \\
\text { ulnar. }\end{array}$ & 11 \\
\hline $\begin{array}{l}\text { Tipo II: Entre los ramos para el } \\
\text { músculo flexor profundo de los dedos, } \\
\text { provenientes de ambos nervios. }\end{array}$ & 35 & $\begin{array}{l}\text { Tipo I C: Comunicación desde el nervio interóseo } \\
\text { anterior hacia el nervio ulnar }\end{array}$ & 31 \\
\hline $\begin{array}{l}\text { Tipo III: Combinación de las } \\
\text { comunicaciones anteriomente } \\
\text { expuestas. }\end{array}$ & 4 & $\begin{array}{l}\text { Tipo II: Combinación doble entre el nervio interóseo } \\
\text { anterior y el nervio ulnar }\end{array}$ & 11 \\
\hline
\end{tabular}


cual se procedió a realizar una detallada disección tanto de la región cubital como del compartimento anterior del antebrazo, utilizando para ello material quirúrgico ad hoc.

Se determinó el punto de origen del NIA y para ello se registró la distancia de este punto respecto a la LBE. Adicionalmente se describió el origen del NIA respecto a los arcos de origen de los músculos pronador redondo y flexor superficial de los dedos.

La disección se inició trazando dos incisiones horizontales, una proximal a la LBE, y otra distal, a nivel de los procesos estiloides del radio y la ulna. En seguida estas incisiones horizontales fueron conectadas por una incisión longitudinal en la superficie anterior del antebrazo. Se procedió a retirar la piel, se prescindió de la fascia superficial, los elementos vasculares de la región cubital y el lacertus fibroso. Una vez disecado el nervio mediano y sus ramos en la fosa cubital (eventualmente también el nervio interóseo anterior), se seccionó el tendón del músculo pronador redondo, para acceder al plano profundo del compartimiento anterior del antebrazo. Se disecó y registró la biometría de los ramos que el NIA otorgó a los músculos del antebrazo. Por último, se revisó la existencia de variantes anatómicas, ya sea, la presencia de ramos comunicantes entre los nervios mediano, NIA y el nervio ulnar, como también, la presencia de la cabeza accesoria del músculo flexor largo del pulgar.
Todas las mediciones fueron realizadas con un caliper digital Mitutoyo ${ }^{\circledR}$ de $0,01 \mathrm{~mm}$ de precisión. Complementariamente las muestras disecadas fueron fotografiadas con una cámara digital Canon ® Rebel XTI.

\section{RESULTADOS}

El NIA estuvo presente en la totalidad de los miembros disecados. La distancia promedio entre su punto de origen y la LBE alcanzó los 46,11 mm con una desviación estándar de 17,54 mm (intervalo desde los 17,94 mm hasta los 86,89 mm, distal a la LBE). En el $97 \%$ de los casos, este ramo surgió en el tercio proximal del antebrazo; en el $58 \%$ de estos, su punto de origen se localizó en la mitad distal del tercio proximal del antebrazo.

En el $37 \%$ de los casos, el NIA se originó en el tramo existente entre el arco formado entre las cabezas de origen del músculo pronador redondo y el arco de las cabezas de origen del músculo flexor superficial de los dedos. Adicionalmente, en un $23 \%$ de los casos, este nervio surgió en un punto proximal a la ubicación de los dos arcos mencionados (Fig. 2).

En la totalidad de los antebrazos analizados el NIA otorgó ramos para los músculos flexor profundo de los de-
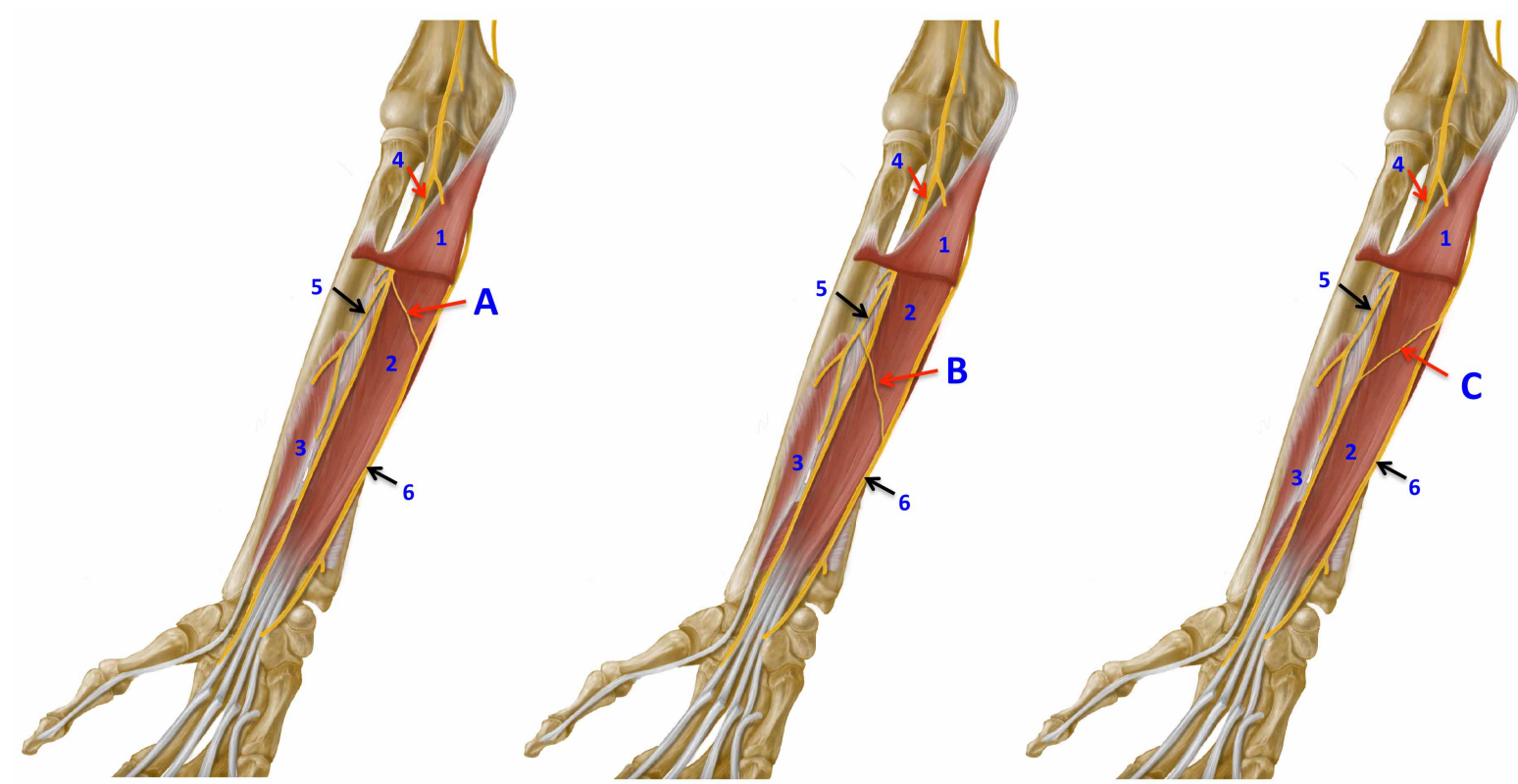

Fig. 1. Vista anterior, antebrazo derecho. 1: Músculo flexor superficial de los dedos, 2: Músculo flexor profundo de los dedos, 3: Músculo flexor largo del pulgar, 4: Nervio mediano, 5: Nervio interóseo anterior, 6: Nervio ulnar. A: Comunicación nervio mediano - nervio ulnar, B: Comunicación nervio interóseo anterior - nervio ulnar, C: Comunicación nervio ulnar - nervio mediano. 


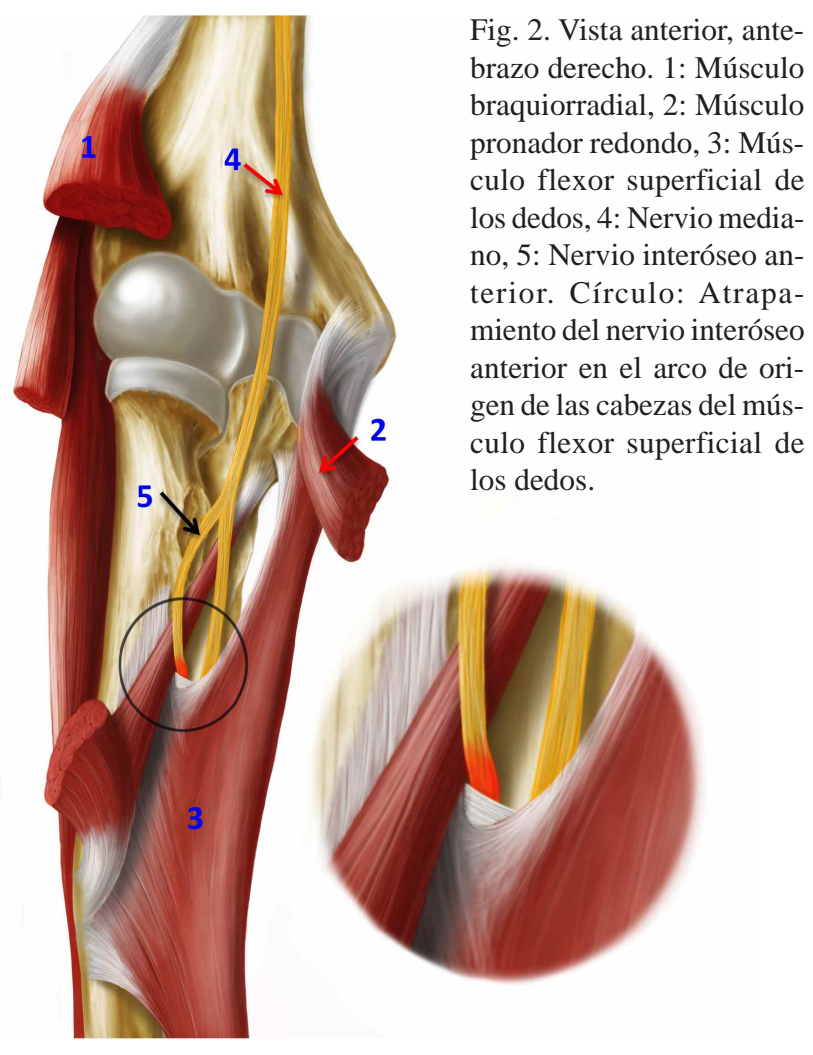

dos y pronador cuadrado. En un $93 \%$ de los casos, este nervio también emitió ramos para el músculo flexor largo del pulgar (Fig. 3A - B).

En 4 casos el NIA suministró ramos para los músculos del plano superficial del compartimiento anterior del antebrazo. En tres de estos, el NIA inervó al músculo flexor superficial de los dedos, y en uno, otorgó ramos a los músculos flexor radial del carpo y palmar largo. anterior también entregó ramos para alguno de los músculos del plano superficial del compartimiento anterior del antebrazo. En tres de estos, este nervio inervó al músculo flexor superficial de los dedos, y en uno, entregó ramos a los músculos flexor radial del carpo y palmar largo.

Respecto a la presencia de variantes anatómicas en un caso se observó ramo comunicante entre el NIA y el nervio ulnar. Su longitud alcanzó los 60,21 mm, el cual presentó una dirección oblicua proximal distal, desde el NIA a 79,55 mm de la LBE, hasta alcanzar el nervio ulnar a $128,42 \mathrm{~mm}$ de la LBE (Fig. 4). En tres antebrazos se encontraron la cabeza accesoria del músculo flexor largo del pulgar, en ellos, esta cabeza se originó en el epicondilo medial del húmero, insertándose en el tendón del músculo flexor largo del pulgar. Estos vientres musculares presentaron aspecto fusiforme con un ancho y longitud promedio de $6,17 \mathrm{~mm}$, y $117,18 \mathrm{~mm}$, respectivamente.
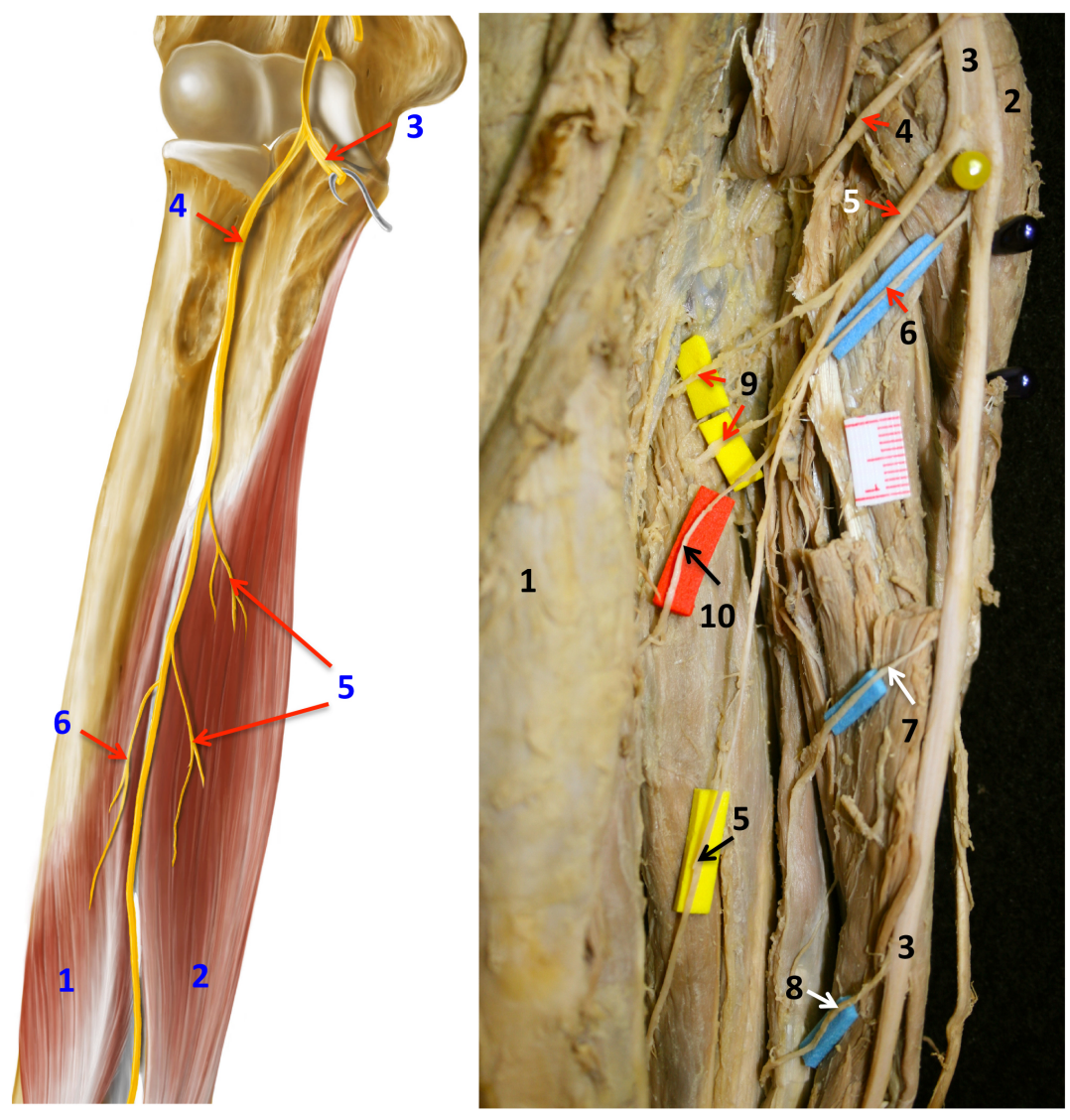

Fig. 3. A. Vista anterior, antebrazo derecho. 1: Músculo flexor largo del pulgar, 2: Músculo flexor profundo de los dedos, 3: Nervio mediano, 4: Nervio interóseo anterior, 5: Ramos motores del músculo flexor profundo de los dedos, 6: Ramo motor del músculo flexor largo del pulgar. B: Vista anterior, antebrazo derecho. 1: Músculo braquiorradial, 2: Músculo pronador redondo, 3: Nervio mediano, 4: Ramo motor para el músculo pronador redondo, 5: Nervio interóseo anterior, 6: Ramo motor R1 para el músculo flexor superficial de los dedos, 7: Ramo motor R2 para el músculo flexor superficial de los dedos, 8: Ramo motor R3 para el músculo flexor superficial de los dedos, 9: Ramos motores del músculo flexor profundo de los dedos, 10: Ramo motor del músculo flexor largo del pulgar. 


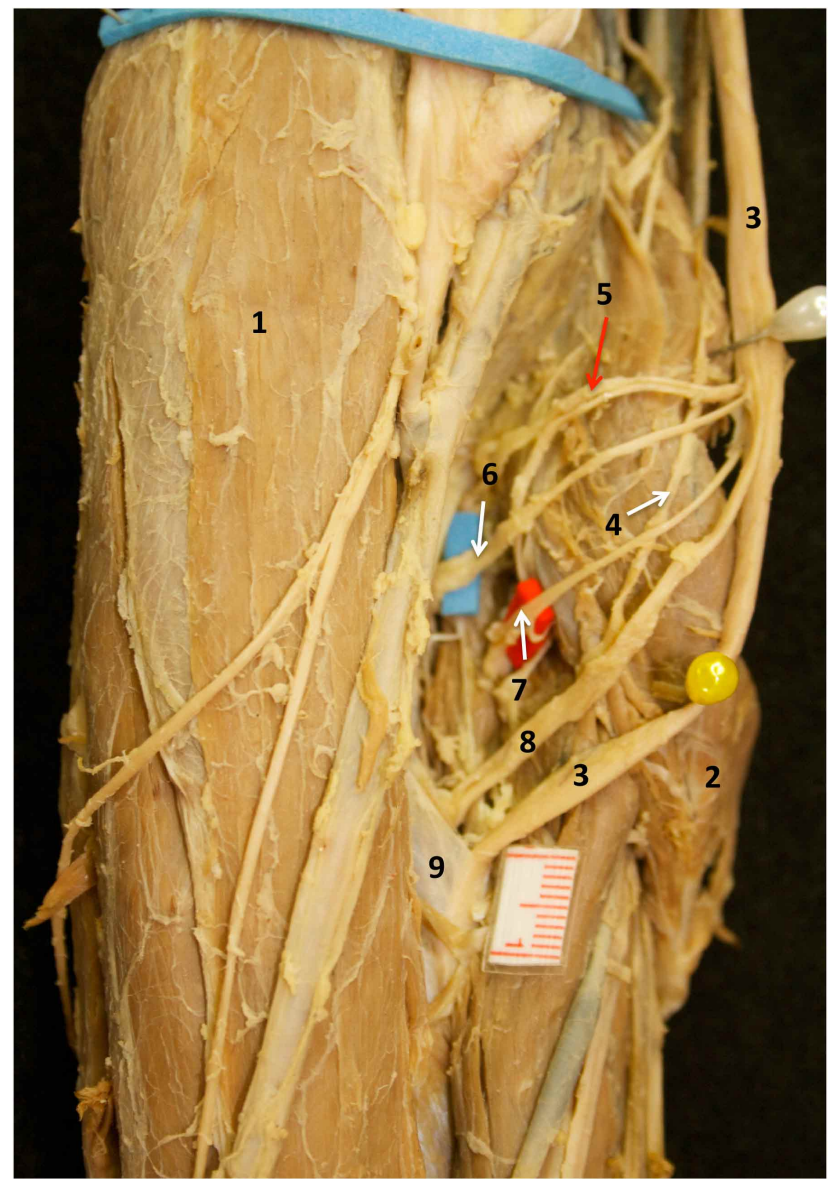

Fig. 4. Vista anterior, antebrazo derecho. 1: Músculo braquiorradial, 2: Músculo pronador redondo, 3: Nervio mediano, 4: Ramo motor R1 para el músculo pronador redondo, 5: Ramo motor R2 para el músculo pronador redondo, 6: Ramo motor del músculo palmar largo, 7: Ramo motor del músculo flexor radial del carpo, 8: Nervio interóseo anterior, 9: Arco fibroso del músculo flexor superficial de los dedos.

\section{DISCUSIÓN}

En relación al punto de origen del NIA, nuestra investigación cifra en aproximados $46 \mathrm{~mm}$, cifra similar a lo descrito por Canovas et al., sin embargo, es proximal si lo comparamos a los $54 \mathrm{~mm}$ reportado por Tubbs et al. (2010), y distal respecto de los $30 \mathrm{~mm}$, señalados por Gunther et al. (1992). Cabe señalar que todas las cifras anteriormente mencionadas quedan incluidas dentro del intervalo de origen registrado en nuestro estudio. Por otra parte, Poirier \& Charpy (1904) señalaron que este ramo se originó en el espacio existente entre la inserción del músculo pronador redondo y la ubicación del arco perteneciente a las cabezas de origen del músculo flexor superficial de los dedos, disposición que se presentó en el $37 \%$ de nuestros casos; en

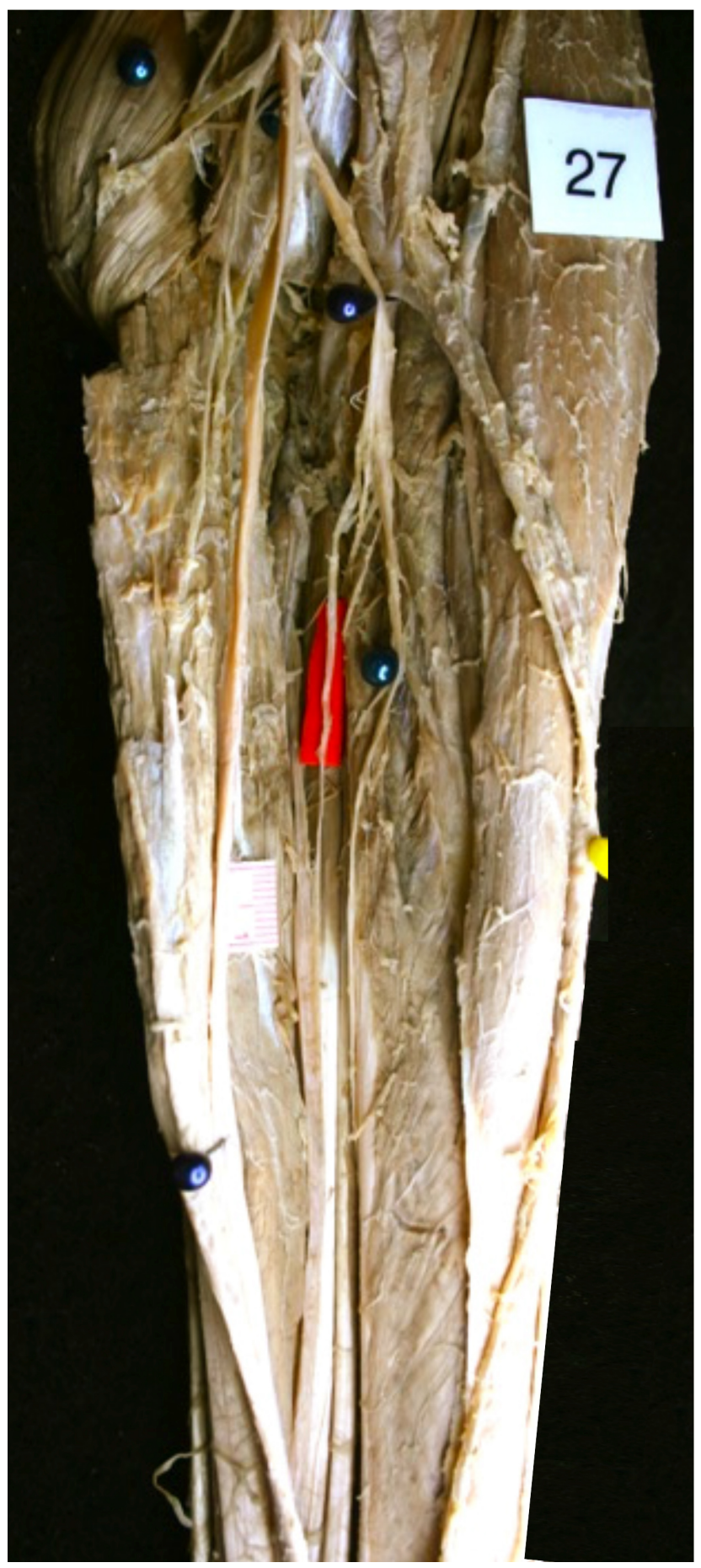

Fig. 5. 1. Músculo braquiorradial, 2: Músculo pronador redondo, 3: Nervio mediano, 4: Nervio interóseo anterior, 5: Nervio ulnar, 6: Ramo comunicante entre los nervios interóseo anterior y ulnar.

cambio el $23 \%$ de los casos, el NIA se originó en un punto proximal a la ubicación de los arcos de los dos músculos vinculados a este espacio. Este dato de ubicación resulta clave al momento de establecer la posibilidad de 
atrapamiento de los nervios mediano y NIA, lo cual representa una referencia al momento de diagnosticar clínicamente los diversos síndromes de atrapamiento.

Respecto de los músculos inervados por el NIA, en la totalidad de los miembros analizados en nuestra investigación, este nervio otorgó ramos para los músculos flexor profundo de los dedos (mitad lateral) y pronador cuadrado. En el $93 \%$ de los casos, también entregó ramos para el músculo flexor largo del pulgar. Estos hallazgos coinciden con lo descrito por Rauber, Linell, Tountas \& Bergman, Williams et al., Drake et al., Schünke et al. y Leversedge et al., quienes señalaron que este nervio es el encargado exclusivo de la inervación de los músculos anteriormente comentados.

Al respecto de esta descripción, Canovas et al. planteó la existencia de una disputa entre autores franceses versus anglosajones y alemanes, los primeros indicaron que el nervio mediano presenta un tronco común para inervar al músculo flexor largo del pulgar, la mitad lateral del músculo flexor profundo de los dedos y un tercer ramo denominado NIA, el cual inerva el músculo pronador cuadrado; en cambio; los segundos plantean que es el NIA se encarga de la inervación de los músculos anteriormente expuestos. A esta divergencia, nuestra investigación agrega el hecho de que en el $13 \%$ de los casos, el NIA entregó ramos para músculos del plano superficial del compartimiento anterior del antebrazo como los músculos flexor radial del carpo, palmar largo y flexor superficial de los dedos, situación anteriormente descrita por autores como Blair \& Joos (1982) y Liu et al. (1997).

En la búsqueda de variantes anatómicas asociadas al trayecto del NIA, Dellon \& Mackinnon y Leversedge et al. describieron la presencia de un fascículo accesorio para el músculo flexor largo del pulgar, el cual estuvo presente en el $45 \%$ de los casos analizados en ambos estudios. Así mismo, Jones et al. (1997) reportaron esta variante muscular en el $30 \%$. Si bien, ambas cifras superan nuestro resultado, queda como premisa de que en el caso de que esta variante esté presente, su disposición generara un arco tendinoso que puede atrapar al NIA o sus ramos para los músculos profundos del compartimiento anterior del antebrazo.

Tountas \& Bergman describieron que este NIA puede recibir, en diferentes sectores de la membrana interósea, ramos comunicantes provenientes tanto del nervio radial, como del nervio interóseo posterior, sin embargo, el ramo comunicante más común en el antebrazo es aquel que comunica al NIA con el nervio ulnar en el punto en que ambos inervan al músculo flexor profundo de los dedos, lo cual, para Leversedge et al. y Llusá et al., se presenta en un 10 a $25 \%$ de la población. Si bien esta cifra es muy diferente al $3 \%$ de casos que se obtuvo en nuestra muestra, es similar a lo resultados obtenidos por Prates et al. (2003), en una serie de 64 antebrazos, en donde este ramo se presentó en un 7,8\%. Por otra parte, Felippe et al. (2012) señalaron que existe la opción de que se presente una comunicación inversa, desde el nervio ulnar hacia el nervio mediano (también denominada comunicación de Marinacci), la cual no estuvo presente en nuestra investigación.

En lo que respecta a las clasificaciones de estos ramos comunicantes, según lo descrito por Nakashima, nuestro caso coincide con el tipo I A, el cual comprende una comunicación desde el NIA hacia el nervio ulnar. Por otra parte, en relación a lo señalado por RodriguezNiedenführ et al., nuestro caso coincidió con el tipo I C. Para Roy et al. la presencia de tales comunicaciones puede causar dificultad en la interpretación de estudios electrofisiológicos, usados para el diagnóstico de neuropatías, lo cual pueden aumentar el riesgo de lesión iatrogénica durante procedimientos quirúrgicos en el antebrazo y la mano.

Por otra parte, un atrapamiento nervioso se describe conceptualmente como la irritación mecánica por la cual un nervio específico se lesiona locamente, en un sitio anatómico vulnerable (Nakano, 1978). Descrito lo anterior y con la intención de analizar la anatomía clínica de los atrapamientos que tanto el nervio mediano como el NIA pueden sufrir en la región proximal del antebrazo, es importante diferenciar los signos y síntomas del síndromes del pronador redondo que afecta al nervio mediano versus el síndrome Kiloh-Nevin, que afecta al NIA. El primero es provocado por fractura, luxaciones, tumores en la región de la articulación del codo, o por la presencia de estructuras que generen una compresión extrínseca del nervio, entre las que destaca, la presencia del ligamento de Struthers (ligamento inconstante que se presenta entre el proceso supraepicondilar y el epicondilo medial del húmero), el excesivo espesor del lacertus fibroso, la presencia de aneurismas, trombosis venosas o trayectos vasculares anómalos (Nigst \& Dick, 1979; Fuss \& Wurzl, 1990; Moore \& Dalley; Schünke et al.; Williams et al.) (Fig. 5).

Entre los hallazgos clínicos relevantes del síndrome del pronador redondo, es importante evaluar la desaparición de la flexión de las articulaciones interfalángicas proximales del segundo y tercer dedo, a lo que se agrega el hecho de que se anula la flexión de las articulaciones interfalángicas distales de los mismos. Si bien no se afec- 
ta la flexión de las articulaciones distales del cuarto y quinto dedos debido (ya que la porción medial del flexor profundo de los dedos es inervada por el nervio ulnar), la capacidad de flexionar las articulaciones metacarpofalángicas del segundo y tercer dedos se altera, ya que los ramos del nervio mediano que inervan el primer y segundo lumbricales se ven comprometidos. Debido a lo anterior, cuando el paciente trata de cerrar el puño, el segundo y tercer dedos quedan parcialmente extendidos, signo conocido como "mano de predicador". A este hallazgo se agrega el signo de la botella, el cual presenta debilidad del músculo abductor corto del pulgar, por lo cual la mano se ve impedida de realizar el cierre de la tapa de una botella. A estos signos, Snell (2006) agregó la debilidad de la flexión de muñeca, la que se acompaña con una desviación ulnar de la mano. Dicha desviación es causada por la parálisis o paresia del músculo flexor radial del carpo, que sumada a la intacta fuerza del músculo flexor ulnar del carpo y de la mitad medial del músculo flexor profundo de los dedos, produce un desbalance muscular que genera la mencionada desviación. Respecto al compromiso sensitivo, debemos destacar que lasomatoestesia de la piel se pierde en la mitad lateral de la palma de la mano, la cara palmar y la parte distal de las superficies dorsales de los tres dedos laterales, sumado a la mitad lateral del dedo anular (Nigst \& Dick).

Comparado con lo anterior, el síndrome del NIA (o de Kiloh-Nevin), presenta debilidad de la pinza digital indice-pulgar, la cual se asocia a la afección del músculo flexor largo del pulgar y el vientre del músculo flexor profundo de los dedos que se dirige hacia el dedo índice. Uno de los signos que se relacionan con esta debilidad, se vincula con la incapacidad de realizar una letra "O" entre los dedos índice y el pulgar. Uno de los elementos que puede ocasionar que la forma de presentación de estos síndromes no sea tan clara, radica en la posibilidad de que el paciente presente ramos comunicantes entre los nervios mediano o NIA con el nervio ulnar o si el origen de los ramos de estos nervios varia en relación al punto de atrapamiento del mismo (Nakano; Williams et al.; Leversedge et al.).

Si relacionamos estas formas de presentación de los diversos síndromes de atrapamiento nervioso en el antebrazo con la presencia de variantes musculares y nerviosas, se genera un escenario que dificulta el correcto diagnóstico de estos síndromes. Producto de ello, estos diagnósticos deben contemplar la necesidad de contar con el apoyo de anatomía por imagen, ya sean, ecografías, tomografías computarizadas o resonancias nucleares magnéticas, las que permitan reconocer no solo la existencia de estas variantes, sino que también faciliten el hallazgo del sitio anatómico del atrapamiento nervioso.
Así mismo, al analizar la forma de presentación clínica de estos síndromes de atrapamiento, siempre se parte de la base del conocimiento explícito del trayecto y distribución de los ramos del nervio mediano y NIA en la fosa cubital, el antebrazo y la mano, sin embargo, los diversos autores, no relatan de forma consensuada un patrón común sobre los puntos de origen o de penetración de los diferentes ramos de los diferentes músculos del antebrazo. Esto no solo dificulta el estudio anatomo-clínico de estos síndromes y su diagnóstico diferencial, sino que también, presentan un obstáculo a la hora de realizar procedimientos invasivos como las transferencias nerviosas, las neurotomías selectivas, los bloqueos nerviosos con toxina botulínica o la aplicación de procedimientos de estimulación eléctrica de los puntos motores (Liu et al.; Olave et al., 2002; Lepage et al., 2005; Riveros et al., 2016).

AGRADECIMIENTOS: Se agradece al ilustrador Sr. Alfredo Torres Parra por sus contribuciones gráficas a esta comunicación científica.

RIVEROS, A.; OLAVE, E. \& SOUSA-RODRIGUES, C. Anterior Interosseous nerve: Course, distribution and clinical implications. Int. J. Morphol., 36(3):1079-1086, 2018.

SUMMARY: The main branch of the median nerve in the forearm is the anterior interosseous nerve (NIA), which innervates most of the muscles of the deep plane of the anterior compartment of the forearm. There are different descriptions about the point of origin and its course, which can determine a potential entrapment of it in its transit through the arcs formed in the heads of origin of the pronator round and flexor digitorum of the fingers muscles, as well as with regard to communicating branches of the NIA with other nerves of the forearm. The aim was to determine the point of origin, course, innervated muscles and presence of communicating branches of the NIA with other nerves of the forearm. 30 forearms of Brazilian adult corpses fixed in formalin belonging to the UNCISAL anatomy laboratory were used, Maceió, Brazil. The point of origin of the NIA reached an average of $46 \mathrm{~mm}$ with a standard deviation of $17.54 \mathrm{~mm}$, distal to the biepicondilar line. In $37 \%$ of the cases, this nerve originated in the existing section of the muscular arches mentioned and in $23 \%$ it arose proximal to the location of these. In all cases, the NIA inervated the flexor digitorum profundus and pronator quadratus and $93 \%$ also inervated the flexor pollicis longus. One case presented a communicating branch between the NIA and the ulnar nerve. In 10 $\%$ of the cases, he presented the variant muscle accessory head of the flexor pollicis longus. All these findings should be considered at the time of correctly diagnosing the different entrapment syndromes that affect both the NIA and the median nerve in the ulnar region and the forearm. Likewise, the success of surgical procedures in these regions is subject to a detailed knowledge of the course and distribution of these nerves.

KEY WORDS: Anatomy; Innervation; Anterior interosseous nerve; Communicating branch; Anatomical variations. 


\section{REFERENCIAS BIBLIOGRAFICAS}

Blair, W. F. \& Joos, K. The innervation of flexor carpi radialis. An interfascicular dissection. Arch. Neurol., 39(10):647-9, 1982.

Canovas, F.; Mouilleron, P. \& Bonnel, F. Biometry of the muscular branches of the median nerve to the forearm. Clin. Anat., 11(4):239-45, 1998.

Comité Federal sobre Terminología Anatómica \& Sociedad Anatómica Española. Terminología Anatómica. Terminología Anatómica Internacional. Buenos Aires, Médica Panamericana, 2001.

Cruveilhier, J. Traitè D`Anatomie Descriptive. 4ª ed. Paris, Asselin, 1851.

Dellon, A. L. \& Mackinnon, S. E. Musculoaponeurotic variations along the course of the median nerve in the proximal forearm. J. Hand. Surg. Br., 12(3): 359-63, 1987.

Felippe, M. M.; Telles, F. L.; Soares, A. C. L. \& Felippe, F. M. Anastomosis between median nerve and ulnar nerve in the forearm. J. Morphol. Sci., 29(1):23-6, 2012.

Fuss, F. K. \& Wurzl, G. H. Median nerve entrapment. Pronator teres syndrome. Surgical anatomy and correlation with symptom patterns. Surg. Radiol. Anat., 12(4):267-71, 1990.

Gunther, S. F.; DiPasquale, D. \& Martin, R. The internal anatomy of the median nerve in the region of the elbow. J. Hand Surg., 17(4):648-56, 1992.

Jones, M.; Abrahams, P. H.; Sañudo, J. R. \& Campillo, M. Incidence and morphology of accessory heads of flexor pollicis longus and flexor digitorum profundus (Gantzer's muscles). J. Anat., 191(Pt. 3):451-5, 1997.

Khale, W. Leonhardt, H. \& Platzer, W. Atlas de Anatomía. Vol. 3. $5^{\text {a }}$ ed. Barcelona, Ediciones Omega, 1994.

Lepage, D.; Parratte, B.; Tatu, L.; Vuiller, F. \& Monnier, G. Extra- and intramuscular nerve supply of the muscles of the anterior antebrachial compartment: applications for selective neurotomy and for botulinum toxin injection. Surg. Radiol. Anat., 27(5):420-30, 2005.

Leversedge, F. J.; Goldfarb, C. A. \& Boyer, M. I. A Pocketbook Manual of Hand and Upper Extremity Anatomy: Primus Manus. Philadelphia, Lippincott Williams \& Wilkins, 2010.

Linell, E. A. The distribution of nerves in the upper limb, with reference to variabilities and their clinical significance. J. Anat., 55(Pt. 2-3):79-112, 1921.

Liu, J.; Pho, R. W.; Pereira, B. P.; Lau, H. K. \& Kumar, V. P. Distribution of primary motor nerve branches and terminal nerve entry points to the forearm muscles. Anat. Rec., 248(3):456-63, 1997.

Llusá, M.; Palazzi, S. \& Valer, A. Anatomía Quirúrgica del Plexo Braquial y de los Nervios Periféricos de la Extremidad Superior. Buenos Aires, Médica Panamericana, 2013.

Moore, K. \& Dalley, A. Anatomía con Orientación Clínica. 4ª ed. Buenos Aires, Médica Panamericana, 2002.

Nakano, K. K. The entrapment neuropathies. Muscle Nerve, 1(4):264-79, 1978.

Nakashima, T. An anatomic study on the Martin-Gruber anastomosis. Surg. Radiol. Anat., 15(3):193-5, 1993.

Nigst, H. \& Dick, W. Syndromes of compression of the median nerve in the proximal forearm (pronator teres syndrome; anterior interosseous nerve syndrome). Arch. Orthop. Trauma Surg., 93(4):307-12, 1979.

Olave, E.; Gabrielli, C.; Braga, M. T. T. \& del Sol, M. Biometric aspects of the motor branches of the musculocutaneous nerve to the brachial muscle. Rev. Chil. Anat., 20(2):231-6, 2002.

Poirier, P. \& Charpy, A. Traité D`Anatomie Humaine. Vol 3. 12 ed. Paris, Masson et Cie, 1904.

Prates, L. C.; Carvalho, V. C.; Prates, J. C.; Langone, F. \& Esquisatto, M. A. M. The Martin-Gruber anastomosis in brazilians: An anatomical study. Braz. J. Morphol. Sci., 20(3):177-80, 2003.

Pró, E. A. Anatomía Clínica. Buenos Aires, Médica Panamericana, 2012.

Rauber, A. Lehrbuch der Anatomie des Menschen. Vol. 2. 5 ${ }^{\mathrm{a}}$ ed. Leipzig, Verlag von Arthur Georgi, 1898.

Riveros, A.; Sousa-Rodrigues, C. F. \& Olave, E. Origin and distribution of the motor branches for the superficial muscles of the anterior region of the forearm in brazilian individuals. Int. J. Morphol., 34(1):356-64. 2016.

Rodriguez-Niedenführ, M.; Vazquez, T.; Parkin, I.; Logan, B. \& Sañudo, J. R. Martin-Gruber anastomosis revisited. Clin. Anat., 15(2):129-34, 2002.

Rouvière, H. \& Delmas, A. Anatomía Humana: Descriptiva, Topográfica y Funcional. Vol. 3. 11 a ed. Barcelona, Masson, 2005.
Roy, J.; Henry, B. M.; Pekala, P.; Vikse, J.; Saganiak, K.; Walocha, J. A. \& Tomaszewski, K. A. Median and ulnar nerve anastomoses in the upper limb: A meta-analysis. Muscle Nerve, 54(1):36-47, 2016.

Sappey, P. C. Traite D`Anatomie Descriptive. Vol. 3. 4a ed. Paris, Lecrosnier et Babé, 1889.

Schünke, M.; Schulte, E. \& Schumacher, U. Prometheus: Texto y Atlas de Anatomia. Buenos Aires, Médica Panamericana, 2005.

Snell, R. Clinical Anatomy by Systems. 6 ${ }^{\mathrm{a}}$ ed. Lippincott Williams \& Wilkin, 2006.

Testut, L. \& Latarjet, A. Tratado de Anatomía Humana. Vol. 3. $9^{\text {a }}$ ed. Barcelona, Salvat, 1972.

Tountas, C. \& Bergman, R. Anatomics Variations of the Upper Extremity. Londres, Churchill Livingstone, 1993.

Tubbs, R. S.; Jones, V. L.; Loukas, M.; Cömert, A.; Shoja, M. M.; Wellons, J. C. 3rd. \& Cohen-Gadol, A. A. Anatomy and landmarks for branches of the brachial plexus: a vade mecum. Surg. Radiol. Anat., 32(3):261-70, 2010.

Williams, P. L.; Bannister, L. H.; Berry, M. M.; Collins, P.; Dyson, M.; Dussek, J. E. \& Ferguson, M. W. J. Anatomía de Gray. Bases Anatómicas de la Medicina y la Cirugía. Vol 2.38 ed. Madrid, Churchill Livingstone-Harcourt Brace, 1998.

\author{
Dirección para correspondencia: \\ Klgo. Mg. Andrés Riveros Valdes \\ Depto. de Ciencias Morfológicas \\ Universidad San Sebastián \\ Sede Concepción \\ Lientur 1457 \\ C.P: 4080871 \\ Concepción \\ CHILE
}

Email: andres.riveros@uss.cl

Recibido : 03-04-2018

Aceptado: 30-06-2018 